\title{
Molecular dynamics simulation of the fragile glass former ortho-terphenyl: a flexible molecule model. II. Collective Dynamics
}

\author{
S. Mossa ${ }^{1,2}$, G. Ruocco ${ }^{2}$ and M. Sampoli ${ }^{3}$ \\ ${ }^{1}$ Center for Polymer Studies and Department of Physics, Boston University, Boston, Massachusetts 02215 \\ 2 Dipartimento di Fisica and Istituto Nazionale di Fisica per la Materia, Università di Roma La Sapienza, \\ P.le Aldo Moro 2, I-00185, Roma, Italy \\ 3 Dipartimento di Energetica and INFM, Università di Firenze, Via Santa Marta 3 , Firenze, I-50139, Italy
}

(November 10, 2018)

\begin{abstract}
We present a Molecular Dynamics study of the collective dynamics of a model for the fragile glass-former orthoterphenyl. In this model, introduced by Mossa, Di Leonardo, Ruocco and Sampoli [S. Mossa, R. Di Leonardo, G. Ruocco and M. Sampoli, Phys. Rev. E 62, 612 (2000)], the intramolecular interaction among the three rigid phenyl rings is described by a set of force constants whose value has been fixed in order to obtain a realistic isolated molecule spectrum. The interaction between different molecules is described by a Lennard Jones site-site potential. We study the behavior of the coherent scattering functions $F_{t}(q, t)$, considering the density fluctuations of both molecular and phenyl rings centers of mass; moreover we directly simulate the neutron scattering spectra taking into account both the contributions due to carbon and hydrogens atoms. We compare our results with the main predictions of the Mode Coupling Theory and with the available coherent neutron scattering experimental data.
\end{abstract}

PACS number(s): 64.70.Pf, 71.15.Pd, 61.25.Em, 61.20.-p

\section{INTRODUCTION}

The understanding of the supercooled liquid and glassy phases in molecular systems is, nowadays, one of the main tasks of the physics of disordered materials (see [2, 3] and references therein for a general review). Two main theories provide us a description of the glass transition respectively from a thermodynamical and dynamical point of view.

The first one (see [4] and references therein) is based on first-principles computation of the equilibrium thermodynamics of glasses and consider the glass transition as a true thermodynamic transition. In this context, the incoming of the glassy state is associated with an entropy crisis, i. e. the vanishing of the configurational entropy of the thermodynamically relevant states.

The second approach is the Mode Coupling Theory (MCT) [5.6] which studies the long-time structural dynamics and its relation with the glass transition; in this context this transition has to be considered not as a regular thermodynamical phase transition involving singularities of some observables, but as a kinetically induced transition from an ergodic to a non-ergodic behavior. The structural dynamics becomes so "slow" that the system of interest appeared frozen on the experimental time scales.

On the experimental side, the collective dynamics of a huge variety of molecular systems has been investigated by means of several experimental techniques; colloids [7, 8$]$ and ortho-terphenyl (OTP) [9 12] are the most widely studied fragile [2] supercooled liquids. They are only examples of an enormous experimental work (the interested reader is referred to Ref. [3] for an accurate comprehensive review). Moreover, in the last ten years the analysis of experimental results has been flanked by extensive use of numerical techniques, mainly Molecular Dynamics (MD) and Monte Carlo (MC) simulations. The almost exponential growth of computational capabilities allows us to reach simulation times of the order of microseconds for simple monoatomics systems and of several tens nanosecond in the case of complex molecular systems; such performances definitely permit comparisons of numerical results with the structural very long time properties of the real systems. We remember, among many others, the results concerning the structural dynamics of two molecular liquids like SPC/E water 13. and OTP [14,15]. We emphasize here the fact that the models involved in these studies are molecular and rigid in the sense that they have a structure and take into account the orientational properties of the molecules but they disregard the role played by internal degrees of freedom on the overall dynamical behavior of the system.

In a recent paper [1] we have introduced a new model for the intramolecular dynamics of orthoterphenyl (OTP) $\left(T_{m}=329 \mathrm{~K}, T_{c} \simeq 290 \mathrm{~K}, T_{g}=243 \mathrm{~K}\right)$, one of the most deeply studied substances in the liquid, supercooled and glassy state. The introduction of such a flexible model allows us to understand the role of internal degrees of freedom in the short time (fast) dynamics 16] taking place on the time scale of a few picoseconds and to study their possible coupling with the long time center of mass dynamics [17]. Moreover would allow us to emphasize once more the universality of the Mode Coupling Theory (MCT) approach for supercooled liquids and, in particular, of its molecular version in the case of complex molecular liquids. In Ref. [1] we introduced in details the in- 
termolecular model and we showed some results based on MD calculations; the self (one particle) dynamical properties of a system composed of 108 molecules have been studied in details and we have found good agreement with the main predictions of the MCT and with the experimental results related to the self intermediate scattering function [18,19] and the self diffusion [20,21]. In the present paper we complete the picture considering the collective highly cooperative structural dynamics controlling the rearrangement of big portions of the system. We describe the dynamics of the molecules at the level of molecular centers of mass and at the level of phenyl rings center of mass. Moreover we calculate the neutron coherent scattering function taking into account both the contributions due to carbon and hydrogen atoms in order to make a direct comparison with the experimental results.

The paper is organized as follows: in Sec. II we summarize the main predictions of the Mode Coupling Theory (MCT) and we give a schematic introduction to the Molecular Mode Coupling Theory (MMCT); in Sec. III we briefly describe the model and we recall some computational details. In Sec. IV we study the temperature and momentum dependence of the collective dynamics of the molecular and of the phenyl rings centers of mass. In Sec. V we make a deep comparison between the experimental results of neutron scattering and the simulated neutron spectra calculated taking into account the interactions with both carbon and hydrogen atoms. Finally, Sec. V1 contains a discussion of the results obtained and some conclusions.

\section{MODE-COUPLING AND MOLECULAR MODE-COUPLING THEORY}

As we have already stressed in Sec. If two main theories, one intrinsically thermodynamical the other purely dynamical [5] 6] have been developed up to now to describe the phenomenology observed in the glass transition. In this paper we will make a comparison of our numerical results with the main predictions of MCT about the center of mass structural relaxation dynamics. The reason for this is twofold: first of all this is, indeed, the theory taking into account the states actually accessible by the system on the time scale of a typical simulation. Moreover, even if some experimental results, like the presence of the so-called knee characterizing the low-frequency behavior of the light scattering susceptibility 22,23 or the presence of a cusp in the non-ergodicity parameter 24, seem to contradict some of its predictions, such theory has been verified to hold for different experimental data on a very wide time region. Finally, in the last two years successful efforts have been made in order to generalize the theory to liquids of rigid molecules of arbitrary shape [25] (see also Ref. [26] for the particu- lar case of a liquid of linear rigid molecules) taking into account both translational and rotational dynamics.

In Ref. [1] we have summarized the main predictions of the so called ideal MCT so that here we recall only the fundamental equations of the theory, concerning the collective intermediate scattering function and that we will use in the following sections. MCT interprets the glass formation as a dynamical transition from an ergodic to a non-ergodic behavior at a cross-over temperature $T_{c}$; the theory is written as a self-consistent dynamical treatment [5] of the intermediate scattering function, i. e. the time correlation of the density fluctuations of momentum $q$.

This theoretical scheme can be considered the mathematical description of the physical picture of the cage effect. Following the dynamics of a tagged particle it is possible to recognize two main dynamical regions. On small time scale of order of some picosecond, (the $\beta$-region) the dynamics of the particle is confined into a limited region (the cage) builded up by the nearest neighbors. In this regime it is possible to write the intermediate scattering function as

$$
F(q, t)=f(q)+h(q) \sqrt{\frac{\left|T-T_{c}\right|}{T_{c}}} G_{ \pm}\left(t / \tau_{\sigma}\right)
$$

where $f(q)$ is the non-ergodicity parameter (also referred to as the Debye-Waller factor), $h(q)$ is an amplitude independent of temperature and time and the \pm in $G_{ \pm}$ corresponds to time larger or smaller with respect to $\tau_{\sigma}$, a parameter that fix the time scale of the $\beta$-process. At this stage, the time dependence of the correlation functions is all embedded in the $q$-independent function $G_{ \pm}$. $G_{ \pm}(t)$ is asymptotically expressed by two power laws, respectively the critical decay

$$
G_{+}\left(t / \tau_{\sigma}\right)=\left(t / \tau_{\sigma}\right)^{-a} \quad \tau_{0}<<t<<\tau_{\sigma}
$$

and the von Schweidler law,

$$
G_{-}\left(t / \tau_{\sigma}\right)=-\left(t / \tau_{\sigma}\right)^{b} \quad \tau_{\sigma}<<t<<\tau_{\alpha} ;
$$

characterized by the temperature and momentum independent exponents $a$ and $b$; here $\tau_{\alpha}$ is the structural relaxation time.

At time scales of order of $\tau_{\alpha}$ the cages start to break down and the particle starts to diffuse approaching pure brownian motion. This long time part of the dynamics is the so called $\alpha$-region and is well described by a stretched exponential function

$$
F(q, t) \simeq f(q) \exp \left\{-\left(\frac{t}{\tau_{\alpha}}\right)^{\beta_{\alpha}}\right\}
$$

which verifies the time-temperature superposition principle (TTSP). The $\alpha$ time scale $\tau_{\alpha}$ depends on temperature trough a power law of the form 


$$
\tau_{\alpha} \propto\left(T-T_{c}\right)^{-\gamma}
$$

The momentum dependence of the dynamical parameters in the collective dynamics case is not trivial as in the single-particle case. When looking at the structural collective dynamics studying different values of the momentum $q$ means studying the highly cooperative time evolution of cages of average dimension $2 \pi / q$; it is clear that such time evolution is strongly coupled to the static topological structure of the system. More precisely, MCT predicts that the parameters $f(q), h^{-1}(q), \beta_{\alpha}$ and $\tau_{\alpha}(q)$ oscillate in phase with the static structure factor $S(q)$.

The result concerning the momentum dependence of the collective relaxation time is quite general and is well known as de Gennes narrowing [27]. A general relation [28] holds among the one-particle $\tau_{s}$ and the collective $\tau_{c}$ relaxation times, namely $\tau_{c}(q) \simeq S(q) \tau_{s}(q)$; if the diffusion limit is appropriate for $F_{s}(q, t)$, i.e. for values of $q$ close to the first peak of the static structure factor, we obtain

$$
\tau_{c}(q, T) \simeq \frac{1}{D(T)} \frac{S(q)}{q^{2}}
$$

where $D(T)$ is the diffusion coefficient at temperature $T$ and $S(q)$ is supposed to be nearly temperature independent.

In summary, the momentum dependence of the collective dynamics is non trivially driven by the static structure of the system. In particular, for molecular systems, the small length scale structure is determined by orientational properties of the single molecules; a pure molecular translational dynamics will, obviously, loose all the dynamical features controlled by the high momentum part of the static structure factor.

An important step toward a correct explanation of the dynamics of molecular systems is to write down a Molecular Mode Coupling Theory (MMCT) [25]26] taking into account both translational and rotational degrees of freedom. If we consider a system of $N$ identical rigid molecules of arbitrary shape described by their center of mass positions $\bar{r}_{j}(t)$ and by the Euler angles $\Omega_{j}(t)=\left(\phi_{j}(t), \theta_{j}(t), \chi_{j}(t)\right)$ we can write the timedependent microscopic one-particle density as

$$
\rho(\bar{r}, \Omega, t)=\sum_{n=1}^{N} \delta\left[\bar{r}-\bar{r}_{n}(t)\right] \delta\left[\Omega, \Omega_{n}(t)\right] .
$$

Expanding with respect to the complete set of functions given by the plane waves and the Wigner matrices $D_{m n}^{l}(\Omega)$, we have the tensorial density modes

$$
\rho_{l m n}(\bar{q}, t)=i^{l}(2 l+1)^{1 / 2} \sum_{n=1}^{N} e^{i \bar{q} \cdot \bar{r}_{n}(t)} D_{m n}^{l *}\left[\Omega_{n}(t)\right] .
$$

Then, the generalization of the intermediate scattering function to the molecular case is the tensorial quantity

$$
S_{l m n ; l^{\prime} m^{\prime} n^{\prime}}(\bar{q}, t)=\frac{1}{N}\left\langle\delta \rho_{l m n}^{*}(\bar{q}, t) \delta \rho_{l^{\prime} m^{\prime} n^{\prime}}^{*}(\bar{q})\right\rangle .
$$

These correlators are directly related to experimental quantities 26]; for $l=l^{\prime}=0$, they describe the dynamics of translational degrees of freedom which can be measured by neutron scattering when looking at the center of mass low frequency part of the spectrum; if the molecules possess a permanent dipolar moment, the correlators with $l=l^{\prime}=1$ give information related to dielectric measurements and $l=l^{\prime}=2$ is finally related to the orientational contribution to light scattering. At this stage, provided the static angular correlators $S_{l m n ; l^{\prime} m^{\prime} n^{\prime}}(\bar{q}, 0)$ and the number density, it is possible to give a closed set of equations for the matrix $\mathbf{S}$ that completely solve the problem of a liquid of rigid molecules.

The problem is that MMCT seems to be not enough for a high structured molecular system like OTP; we will see that it is not possible to explain some features of the momentum dependence of the structural dynamics without taking into account the internal degrees of freedom (i. e. rotations of the side rings with respect to the central one) that turn out to be strongly coupled to the long time behavior of the density fluctuations.

\section{MODEL AND COMPUTATIONAL DETAILS}

In this section we give a brief description of the model and we address the reader to Ref. [1] for details.

In our model, the OTP molecule is constituted by three rigid hexagonal rings of side $L_{a}=0.139 \mathrm{~nm}$ representing the phenyl rings; two adjacent vertices of the central ring are bonded to one vertex of the two lateral rings by bonds of equilibrium length $L_{b}=0.15 \mathrm{~nm}$. In the isolated molecule equilibrium position, the two lateral rings lie in planes that form an angle of about $54^{\circ}$ with respect to the central ring's plane. In the model the two lateral rings are free to rotate along the molecular bonds, to stretch along the bonds and to tilt out of the plane identified by the central ring. The intramolecular potential is then written as a sum of harmonic and anharmonic terms each one controlling one of these features. Every term is multiplied by a coupling constant whose actual value is determined in order to have a realistic isolated molecule vibrational spectrum. The intermolecular interaction is of the site-site Lennard-Jones type; each of these sites corresponds to a vertex of a hexagon and is occupied by a fictious atom of mass $M_{C H}=13$ amu representing a carbon-hydrogen pair. The actual values of the parameters $\sigma_{L J}$ and $\epsilon_{L J}$ have been fixed in order to have the first maximum of the static structure factor $S(q)$ in the experimentally determined position [29] and to obtain the correct diffusional properties 2021]; the cut-off has been fixed to the value $r_{c}=1.6 \mathrm{~nm}^{-1}$. It is worth noting 
here that obviously the parameterization of the potential cannot be perfect; in our case it is possible to reproduce quite well the experimental results on the whole investigated temperature range shifting the MD thermodynamical points at temperatures of $20 \mathrm{~K}$ above their true values. The MD simulated system is composed of 108 molecules (324 phenyl rings for a total of 1944 Lennard Jones interaction sites); at each time step the intramolecular and intermolecular interaction forces are calculated and the equation of motion for the rings are solved for the translational and rotational part separately.

Wide temperature and momentum ranges have been investigated for values of temperature $380 \leq T \leq 440 \mathrm{~K}$ and momentum $2 \leq q \leq 30 \mathrm{~nm}^{-1}$ (the runs details are shown in Table III of Ref. [1]) and the total simulation time is of almost one hundred nanoseconds.

\section{MOLECULES AND PHENYL RINGS}

The collective density fluctuations dynamics is embedded in the coherent intermediate scattering function in general defined as

$$
F_{t}(q, t)=\frac{1}{N S(q)}\left\langle\sum_{i=1}^{N} \sum_{j=1}^{N} e^{-i \bar{q} \cdot\left[\bar{x}_{i}(t)-\bar{x}_{j}(0)\right]}\right\rangle
$$

where $N$ is the number of molecules involved and $S(q)$ the static structure factor. In the present case the position variables $x_{k}(t)$ can be identified with different quantities; here we are interested in the dynamics of the molecular centers of mass and of the phenyl rings so that we will consider the following scattering functions

$$
\begin{aligned}
& F_{t}^{(M)}(q, t)=\frac{1}{N_{M} S^{(M)}(q)}\left\langle\sum_{\xi^{\prime} \xi^{\prime \prime}} e^{-i \bar{q} \cdot\left[\bar{M}_{\xi^{\prime}}(t)-\bar{M}_{\xi^{\prime \prime}}(0)\right]}\right\rangle \\
& F_{t}^{(R)}(q, t)=\frac{1}{N_{R} S^{(R)}(q)}\left\langle\sum_{i j} \sum_{\xi^{\prime} \xi^{\prime \prime}} e^{-i \bar{q} \cdot\left[\bar{R}_{i \xi^{\prime}}(t)-\bar{R}_{j \xi^{\prime \prime}}(0)\right]}\right\rangle
\end{aligned}
$$

Here $\bar{M}_{\xi^{\prime}}(t)$ is the position of the center of mass of the molecule $\xi^{\prime}$ at time $t\left(\xi^{\prime}=1 \ldots N_{M}\right), \bar{R}_{i \xi^{\prime}}(t)$ the position of the center of mass of the phenyl ring $i(i=1 \ldots 3)$ pertaining to the molecule $\xi^{\prime}$; the functions are renormalized to the corresponding static structure factors. From now on, the superscripts (R) and (M) will be referred to molecular and rings quantities respectively.

As in the case of the incoherent scattering function, at every temperature investigated we have reconstructed the whole curve, even on very short time scales, by mean of two sets of system configuration campionated with different frequencies (see Table III of Ref. [1]). At every investigated temperature, we considered the momentum values $q_{1}=14 \mathrm{~nm}^{-1}$ and $q_{2}=19 \mathrm{~nm}^{-1}$ corresponding to the first and second peak of the experimental static structure factor, averaging on the values of $q$ falling in the interval $q \pm \Delta q$ with $\Delta q=0.2 \mathrm{~nm}^{-1}$. Moreover, the momentum dependence of the principal dynamical parameters has been investigated at $T=280,300,330 \mathrm{~K}$ for values of momenta ranging from 2 to $30 \mathrm{~nm}^{-1}$.

We made a long time analysis in term of the usual stretched exponential form Eq. (雨) determining the temperature and momentum dependence of the fitting parameters $\tau_{\alpha}, \beta_{\alpha}$ and $f_{q}$ and verifying the TTSP.

In Fig. 1 1 we show $F_{t}^{(R)}(q, t)$ calculated at $q_{1}$ for the temperatures $T=280,300,320,350,370,390,410,430 \mathrm{~K}$ (from top to bottom); as in the case of the self dynamics, every curve decays to zero in the considered time window and it is clearly visible the two steps decaying pattern. The long time part of these $\left.F_{t}^{(} R\right)$ have been fitted to Eq. (4) and the parameters $\tau_{\alpha}, \beta_{\alpha}$ and $f_{q}$ are determined by a least square fitting routine. In the inset we plot the same curves as a function of the rescaled time $\bar{t}=t / \tau_{\alpha}^{(R)}$; all the curves collapse pretty well on a single master curve as predicted by the TTSP. The temperature dependence of the non-ergodicity parameter $f_{q}^{(R)}$ (top panel) and of the stretching parameter $\beta_{\alpha}^{(R)}$ (bottom panel) are shown in Fig. 2; they are temperature independent, as predicted by MCT. The mean values $f_{q}^{(R)}=0.78$ and $\beta_{\alpha}^{(R)}=0.83$ (dashed line) have to be compared with the values determined in the case of the self dynamics $f_{q} \simeq 0.7$ and $\beta_{\alpha} \simeq 0.8$ [1]. The two values of $\beta_{\alpha}$ are equal in the limit of the error bars; at variance, the value for $f_{q}^{(R)}$ in the collective case is greater than the value found in the one particle case.

In Fig. 3 we plot the structural relaxation times at $q_{1}$ and $q_{2}$ for molecules (triangles and diamonds) and phenyl rings (circles and squares) in order to test if both $\tau_{\alpha}^{(M)}$ and $\tau_{\alpha}^{(R)}$ follow the same power law which is supposed to be momentum independent. Both sets of data have found to be consistent with a power law of the form Eq. (5) with parameters $T_{c} \simeq 268, \gamma \simeq 2.3$; these values have to be compared with the results concerning the one particle dynamics $T_{c}=276 \pm 7 \mathrm{~K}$ and $\gamma=2.0 \pm 0.4$ [1]. The inset show the data plotted as a function of $\bar{T}=T-T_{c}$, in order to stress the power law dependence, and rescaled by an arbitrary factor in order to maximize the overlap.

We now consider the momentum dependence of the collective dynamics at few selected temperatures $T=$ $280,300,330 \mathrm{~K}$, spanning the momentum region in the interval $2 \div 30 \mathrm{~nm}^{-1}$. We test the long time dynamics in term of the stretched exponential function and we verify the MCT predictions on the von Schweidler time region, characterized by the power exponent $b$.

In Fig. 团 (top panel) we show the stretched exponential fits (solid lines) to $F_{t}^{(R)}$ at some selected values of $q$; they works pretty good at least for time values greater than 5 picoseconds as is more clear looking at the bottom panel. Here we show the whole calculated curve (circles) up to $t \simeq 0.05$ ps at $q=14 \mathrm{~nm}^{-1}$ and $T=300 \mathrm{~K}$ together with 
the stretched exponential fit (solid line). From the top panel of Fig. 1 is qualitatively clear that the relaxation time depends non trivially on the momentum values. As we reminded in Sect. II, MCT predicts that in the collective case, at fixed temperature, the relaxation times oscillate in phase with the static structure factor, i. e. they are strongly coupled to the static structure of the system.

In Fig. 5 and Fig. 6 we plot the momentum dependence of the collective relaxation times (top panels) for molecules and rings respectively, at $T=280,300,330 \mathrm{~K}$ (circles, squares and triangles respectively) together with the corresponding static structure factors divided by $q^{2}$ (bottom panels). The correlation among these two quantities, predicted by Eq. (6), is evident; it is also evident that, at variance with the temperature dependence, the momentum dependence of the relaxation times is completely different in the two cases.

In the molecular case (Fig. 5) only a maximum at $q \simeq 9 \mathrm{~nm}^{-1}$ corresponding to intermolecular correlations is present ( a small shoulder at $q \simeq 14 \mathrm{~nm}^{-1}$, related to correlations between rings pertaining to different molecules, can be also identified). In the case of the phenyl rings (Fig. 6) the momentum dependence is much more structured and three main features are present:

(i) a maximum at $q \simeq 9 \mathrm{~nm}^{-1}$ related to correlations between molecular centers of mass

(ii) a shoulder at $q \simeq 14 \mathrm{~nm}^{-1}$ (well developed in a maximum at the lowest temperature $T=280 \mathrm{~K}$ ) related to correlations between rings belonging to different molecules

(iii) a maximum at $q \simeq 22 \mathrm{~nm}^{-1}$ related to correlations between rings pertaining to the same molecules; this is the more important features related mainly to the orientation of the lateral rings with respect to the central ring.

It is clear from this result that in complex molecular glass formers there are intramolecular rotational and vibrational degrees of freedom that couple to the translational long-time modes as already stressed in Ref. [11]; a theory not taking into account these degrees of freedom cannot explain the whole momentum dependence of the centers of mass dynamics.

In Fig. 7 we show the different relaxation times (left panel for molecules and right panel for rings) at the three selected temperatures multiplied by the corresponding diffusion coefficients from Ref. [1]; as predicted by Eq. (6), these products are temperature independent for values of $q$ close to the first maximum of the static structure factor.

In Fig. 8(A) we plot the momentum dependence of the stretching parameter $\beta_{\alpha}^{(R)}$ while in Fig. 8(B) we show the non ergodicity parameter $f_{q}^{(R)}$ at the three selected temperatures together with $S^{(R)}(q) / q^{2}$ ( Fig. 8(C)); also in this case a strong correlation between the oscillations of the quantities is clear, as expected.

We have seen in Sec. II that the long time limit of the $\beta$-region can be described by the von Schweidler power law Eqs. (11) (3). The exponent $b$ is expected to be momentum independent and to assume the same value of the self dynamics case namely $b=0.52$ [18; ; the contrary, the amplitude $h(q)$ is expected to be momentum dependent and to oscillate out of phase with the static structure factor. We then calculated a power law fit in the form $F_{t}^{(R)}(q, t)=f_{q}^{(R)}-c_{2}^{(R)}(q) t^{b^{(R)}}$ for all values of momentum considered, in a time window depending on the particular q-value but always included in the interval $2 \div 30 \mathrm{ps}$; moreover we considered the three parameters free as in the case of the self dynamics. All the observations done in the previous work concerning the great uncertainties on the estimated values of the fitting parameters, hold in the present case. In Fig. 9 (A) we plot the power exponent $b(q)$ that is supposed to be momentum independent; some smooth oscillations are nevertheless present but this can be due to the interplay during the fitting procedure with the other oscillating parameters. In Fig. 9(B) we plot $f_{q}^{(R)}$; these points must be compared with the results of Fig. 8 (B) and the agreement looks quite nice as expected. The value of the plateau, indeed, must be the same if determined as the small time limit of the $\alpha$-region or the long time limit of the $\beta$-process. In Fig. $8(\mathrm{C})$ we plot the quantity $1 / c_{2}^{(R)}(q)$ and also in this case oscillations in phase with $S^{R}(q) / q^{2}$ are evident, as expected.

\section{NEUTRON SCATTERING}

Neutron scattering is one of the most powerful tools used in the study of supercooled liquids and glasses in the $q$-region covered by the MD simulations. Experimentally the scattering function $F_{t}(q, t)$ of Eq. (10) can be determined 10] by neutron scattering experiments either directly, on neutron spin echo instruments, or Fourier transforming the dynamical structure factor $S(q, \omega)$

$$
\frac{S(q, \omega)}{S(q)}=\frac{1}{2 \pi \hbar} \int d t e^{-i \omega t} F_{t}(q, t)
$$

calculated by means of triple axis backscattering or time of flight spectroscopy. The experimental neutron scattering cross section $(d \sigma / d \Omega d E)$ is generally composed of a coherent and an incoherent part

$$
\begin{aligned}
d \sigma / d \Omega d E & \simeq<b>^{2} S_{c o h}(q, \omega) \\
& +\left[<b^{2}>-<b>^{2}\right] S_{i n c o h}(q, \omega)
\end{aligned}
$$

where $b$ is the scattering length and the symbol \langle\rangle denotes an average over the distribution of nuclear spins and isotopes. The isotopic composition of the sample allows us to study selectively the collective motion via coherent scattering from deuterated samples (the scattering 
lengths of $\mathrm{D}$ and $\mathrm{C}$ atoms are basically coincident) and the one particle motion via incoherent scattering from protonated samples.

The interaction of neutrons with a bulk sample of OTP can be simulated numerically taking into account the interactions of neutrons with both carbon (C) and deuterium (D) atoms. $\mathrm{H}$ atoms are not considered in our dynamics but is a reasonable approximation to put them in fixed position on the line extending from the center of the ring trough a carbon atom at the fixed $\mathrm{C}-\mathrm{H}$ distance $d_{C-H}=0.107 \mathrm{~nm}$; so that, knowing the coordinates of the rings, is trivial to reconstruct their own positions. We then define a neutron $(\mathrm{N})$ coherent scattering function $F_{t}^{(N)}(q, t)$ as

$$
\begin{aligned}
F_{t}^{(N)}(q, t)= & \frac{1}{N_{A} S^{(N)}(q)}\left\langle\sum_{\lambda^{\prime} \lambda^{\prime \prime}} \sum_{i j} \sum_{\xi^{\prime} \xi^{\prime \prime}}\right. \\
& \left.b_{\lambda^{\prime}} b_{\lambda^{\prime \prime}} e^{-i \bar{q} \cdot\left[\bar{r}_{\lambda^{\prime} i \xi^{\prime}}(t)-\bar{r}_{\lambda^{\prime \prime} j \xi^{\prime \prime}}(0)\right]}\right\rangle
\end{aligned}
$$

where $N_{A}=N_{C}+N_{H}$ (3456 in this case) is the total number of atoms, $\bar{r}_{\lambda^{\prime} i \xi^{\prime}}(t)$ is the position of the atom $\lambda^{\prime}$ pertaining to the ring $i$ in the molecule $\xi^{\prime}$ and $S^{(N)}(q)$ is the static structure factor of Fig. 11 of Ref. [1]. The number of hydrogen atoms is 4 for each central ring and 5 for each lateral ring. The scattering lengths $b_{\lambda}$ are in principle different for the carbon and deuterium atoms but, as we observed in Ref. [1] , they are both positive and of the same magnitude so that is a good approximation to consider the product $b_{\lambda^{\prime}} b_{\lambda^{\prime \prime}}$ an ineffective positive constant. The function $F_{t}^{(N)}(q, t)$ is the quantity directly comparable with the experimental data.

In the present section we present a comparison between the temperature and momentum dependencies of the MD and experimental spectra of Ref. [12] calculated from perdeuterated $C_{18} D_{14}$ by means of coherent neutron time-of-flight and backscattering spectroscopy.

At this stage few remarks must be done about the momentum dependence of the MD and experimental sets of data. All these data are supposed to depend on the structure of the systems so that, in general, some differences are expected (experimental and MD structures are slightly different [1]). Anyway, two observations about the experimental results must be made. First of all, as reported in Ref. [12], some reservation is necessary for the experimental data at the smallest momenta, $q \leq 6 \mathrm{~nm}^{-1}$, where $f_{q}$ tends toward 1 . Indeed, in this region one expects significant background from incoherent scattering, which contributes about $15 \%$ of the total cross section, and from multiple scattering. Moreover the technique used to determine the values of the dynamical parameters from the experimental data are quite different with respect the MD computation. Indeed, due to the limited dynamical window of the available spectrometers, in the experimental case a direct fit of the data to the stretched exponential function with three independent parameters is not possible. Based on the observations that $\tau(q) \propto \eta(T) / T(\eta(T)$ is the viscosity at temperature $T$ ) and that the line shape is independent on temperature, at fixed momentum $q$ the spectra at different temperatures are rescaled in time to $\bar{t}=t / t_{s}$ where the scaling time is given by $t_{s}=\tau_{\eta}(T) / \tau_{\eta}(T=290 K)$ and $\tau_{\eta}=\eta(T) / T$. In this way the data converge toward a temperature-independent long time asymptote; this is the curve actually fitted to the stretched exponential.

In Fig. 10 we show the temperature dependence of the stretched exponential fitting parameters for the $\alpha$-region of both MD (open circles) and experimental (solid circles) data determined at $q=14 \mathrm{~nm}^{-1}$. In panel (A) we show the structural relaxation times; the inset shows the same data but the MD points have been shifted of $20 \mathrm{~K}$ as already explained in Ref. [1] . We stressed out, indeed, that a non perfect parameterization of the diffusive behavior of the model system controlled by the value of $\epsilon_{L J}$, has shifted the MD thermodynamical point about $20 \mathrm{~K}$ above the corresponding experimental temperature. From the figure is clear that the agreement among the two sets of data is very good on a very wide time region. In panel (B) we show our results for the non ergodicity parameter $f_{q}$ and also in this case the agreement is very good among the two sets of data; in panel (C) we plot the stretching parameters $\beta_{\alpha}$. In this case the MD points are systematically above the experimentally determined data. This effect can be due to the observations made before; moreover, for the determination of the $\beta_{\alpha}$ parameter, the very long time points are crucial and they seem to lack in the experimental data analysis.

With Fig. 11 we start the comparison of the momentum dependence of the two sets of data. In the top panel of Fig. 11 we plot the simulated scattering functions $F_{t}^{(N)}(q, t)$ (symbols) at $T=290 \mathrm{~K}$ at the indicated $q$ together with the stretched exponential long time fits (solid lines). In this case $f_{q}^{(N)}$ is expected to be momentum dependent so that, in order to have a master plot (bottom panel of Fig. 11), we have to show $\bar{F}_{t}^{(N)}(q, t)=F_{t}^{(N)}(q, t) / f_{q}^{(N)}$ as a function of the rescaled time $\bar{t}=t / \tau_{\alpha}^{(N)}$. The rescaled curves collapse quite well on a master curve as expected. In the next three figures we show the comparison for the momentum dependence of the MD data at the three temperatures $T=280,290,300 \mathrm{~K}$ and the experimental data at $T=313,320,330 \mathrm{~K}$; the two sets of temperatures should be comparable due to the $20 \mathrm{~K}$ shift of the MD data.

In Fig. 12 we show the MD (left panel) and experimental (right panel) relaxation times together with the corresponding static structure factors renormalized to $q^{2}$. Striking similarities are clear even if maxima on MD results correspond to bumps of experimental results. The clearest difference is the decrease of $\tau_{\alpha}^{(N)}$ at small value of $q$ that appear to be completely absent in the experimental case. This could be due to the incoherent background on 
the experimental data stressed above; moreover, is well known that sometimes MD is not able to determine the correct value of $\tau$ at small value of $q$ but usually it tends to overestimate its correct value, at variance of this case. Anyway, the agreement among the two sets of data at the higher values of $q$ corresponding to the intramolecular correlations is surprising; none rigid model could be able to do that.

In Fig. 13 we show the results concerning the non ergodicity parameter $f_{q}^{(N)}$. The different curves are temperature independent as expected; both sets of data show a maximum at $q \simeq 14 \mathrm{~nm}^{-1}$, while the maximum at $q \simeq 9$ $\mathrm{nm}^{-1}$ for the MD data correspond to a little shoulder in the experimental case. Also in this case a decreasing part at small $q$ is present in the MD data at variance with the experimental results. But according to Fig. 7 of Ref. 11] the calculation of the non-ergodicity parameter fitting the $\beta$ - region gives a plateau at $q \simeq 6 \mathrm{~nm}^{-1}$ at variance with the increasing behavior of the $\alpha$ - region analysis. However, as already stressed in Sec. IV, these data are supposed to coincide. Concluding, also in this case, the experimental data at small $q$ seem to be not reliable.

In Fig. 14 we finally show the stretching parameter $\beta_{\alpha}^{(N)}$. MD data are very noisy, nevertheless it is possible to recognize an oscillatory behavior; moreover, the points at the smallest available value of $q$ seem to catch the decreasing behavior of the experimental data.

\section{SUMMARY AND CONCLUSIONS}

In this paper we have concluded the analysis of the long time center of mass dynamics of the intramolecular model for OTP introduced in Ref. [1]; there we found good agreement among the diffusion properties of the simulated and the real system as well as among the two single-particle dynamics. Moreover in Ref. [1] we found a very good agreement with the main predictions of MCT. In the present paper we definitely confirm such an agreement.

We have studied the collective density fluctuations on a large temperature and momentum range range, considering both the fluctuations related to molecular and phenyl ring centers of mass. With respect to the temperature dependence we found the usual double step decaying pattern and we confirmed the main predictions of the MCT about the behavior of the stretched exponential parameters; in particular the relaxation times obey the same power law. The momentum dependence of the stretching parameters appears really interesting; MCT predicts that the behavior in the momentum space of the structural relaxation time, at variance with the the trivial square law of the one-particle case, is driven by the structure, namely it is proportional to $S(q) / q^{2}$. In both cases, molecules and phenyl rings, this prediction is completely fulfilled. The phenyl rings behavior is particularly interesting; every feature of the quantity $S(q) / q^{2}$ is mirrored on the $\tau_{\alpha}^{(R)}(q)$ curve at the three investigated temperatures $T=280,300,330 \mathrm{~K}$ up to a value of momentum $q \simeq 30 \mathrm{~nm}^{-1}$. In particular, the maximum at $q \simeq 22 \mathrm{~nm}^{-1}$ is related to fluctuations taking place on molecular length scales; the long time structural dynamics appears, indeed, coupled to the dynamics of internal molecular degrees of freedom. Similar oscillations are present for the other stretched parameters and also the correct behavior for the short time $\beta$-region is found.

The next step has been a comparison among the experimental and simulated neutron scattering spectra calculated considering both the scattering from carbon and deuterium atoms. The temperature dependences of the relaxation times in both cases are well described by the same power law for almost three decades in time; a good agreement is also found for the other stretching parameters. The momentum dependence is qualitatively very similar in both cases; although some different features not clear are present.

At this point some conclusions can be drawn about the capability of our model to describe the long time dynamics of the real system. All the center of mass time scales calculated by molecular dynamics have been found to be consistent with a power law, although some discrepancies were present for the actual value of the power exponent mainly due to uncertainties on the fitting procedure. Moreover it has been shown that our actual MD thermodynamical point is shifted by about $20 \mathrm{~K}$ with respect to the corresponding experimental point. Taking into account all these informations, we plot in Fig. 15 all the time scales related to the centers of mass dynamics considered up to now, both experimental and numerical, as a function of the rescaled temperature $\tilde{T}=T-T_{c}$ where $T_{c}=290 \mathrm{~K} 18$ for the experimental points and $T_{c}=270$ $\mathrm{K}$ for the MD results. In particular, $\tau_{V-F}(T)$ (circles) is the time scale related to the shear viscosity $\eta_{s}$ of Ref. [30], $\tau_{\alpha}(T)$ (left triangles) is the neutron scattering structural relaxation time of Ref. [12] at $q=14 \mathrm{~nm}^{-1}$ and the inverse of the experimental diffusion coefficient (squares) is from Refs. 20] 21]. The MD one-particle relaxation time calculated on phenyl rings $\tau_{s}^{(R)}(T)$ (diamonds) and self-diffusion coefficient (up triangles) are from Ref. [1]; finally, $\tau_{\alpha}^{(R)}(T)$ (down triangles) is the structural relaxation time of Fig. 3 calculated at $q=14 \mathrm{~nm}^{-1}$ on phenyl rings and $\tau_{\alpha}^{(N)}(T)$ the simulated neutron scattering relaxation time of Fig. 10. All the data collapse pretty well for almost three decades in time on the same line corresponding, on a double-log scale, to a power law of the form of Eq. (5) of exponent $\gamma=2.55$ [18].

Concluding, our model has found to be a very successful model for the centers of mass dynamics of the real system, showing a critical behavior consistent with the experimental results in a wide time window. The imple- 
mentation of an intramolecular dynamics is relevant to such extent; in particular, the internal degrees of freedom appear to be strongly coupled to the long time structural dynamics as is also clear from the study of the rotational properties of the system 31. The next step will be the understanding of the role played by the internal degrees of freedom in the fast relaxations observed experimentally $\sqrt{17}$.

\section{ACKNOWLEDGMENTS}

The authors wish to thank F. Sciortino for very useful discussions and J. Wuttke for the raw experimental data of Ref. [12].

[1] S. Mossa, R. Di Leonardo, G. Ruocco, and M. Sampoli, Phys. Rev. E 62, 612 (2000).

[2] C. A. Angell, Science 267, 1924 (1995).

[3] C. A. Angell, K. L. Ngai, G. B. McKenna, P. F. McMillan, and S. W. Martin, J. Appl. Phys. 88, 3113 (2000).

[4] M. Mézard and G. Parisi, Phys. Rev. Lett. 82, 747 (1999); M. Mézard and G. Parisi, J. Phys.: Condens. Matter 12, 6655 (2000).

[5] W. Götze, in Liquids, Freezing and the Glass Transition, edited by J. P. Hansen, D. Levesque and J. ZinnJustin (North-Holland, Amsterdam, 1991); W. Götze and L. Sjörgen, Rep. Prog. Phys. 55, 241 (1992); W. Götze, J. Phys.: Condensed Matter, 11, A1 (1999).

[6] R. Schilling, in Disorder Effects on Relaxational Processes edited by A. Richert and A. Blumen (Springer Verlag, 1994); W. Kob, in Experimental and Theoretical Approaches to Supercooled Liquids: Advances and Novel Applications edited by J. Fourkas et al. (ACS Books, Washington, 1997).

[7] W. van Megen and S. M. Underwood, Phys. Rev. Lett. 70, 2766 (1993); W. van Megen and S. M. Underwood, Phys. Rev. E 49, 4206 (1994).

[8] P. N. Segrè, S. P. Meeker, P. N. Pusey, and W. C. K. Poon, Phys. Rev. Lett. 75, 958 (1995); P. N. Segrè and P. N. Pusey, Phys. Rev. Lett. 77, 771 (1996).

[9] Y. Hwang and G. Q. Schen, J. Phys.: Condens. Matter 11, 1453 (1999).

[10] E. Bartsch, F. Fujara, J. F. Legrand, W. Petry, H. Sillescu and J. Wuttke, Phys. Rev. E 52, 738 (1995); E. Bartsch, F. Fujara, J. F. Legrand, W. Petry, H. Sillescu and J. Wuttke, Phys. Rev. E 53, 2011 (1996).

[11] A. Tölle, H. Schober, J. Wuttke and F. Fujara, Phys. Rev. E 56, 809 (1997).

[12] A. Tölle, J. Wuttke, H. Schober, O. G. Randl and F. Fujara, Eur. Phys. J. B 5, 231 (1998).

[13] F. Sciortino, L. Fabbian, S. H. Chen, and P. Tartaglia, Phys. Rev. E 56, 5397 (1997); F. W. Starr, F. Sciortino, and H. E. Stanley, Phys. Rev. E 60, 6757 (1999); C. Y. Liao, F. Sciortino, and S. H. Chen, Phys. Rev.
E 60, 6776 (1999); F. Sciortino, Chem. Phys. 258, 307 (2000).

[14] L. J. Lewis and G. Wahnström, Phys. Rev. E 50, 3865 (1994); F. Sciortino and P. Tartaglia, J. Phys.: Condens. Matter 11, A261 (1999).

[15] A. Rinaldi, F. Sciortino, and P. Tartaglia, submitted to Phys. Rev. E, cond-mat/0012415.

[16] G. Monaco, D. Fioretto, C. Masciovecchio, G. Ruocco and F. Sette, Phys. Rev. Lett. 82, 1776 (1999); G. Monaco, S. Caponi, R. Di Leonardo, D. Fioretto and G. Ruocco, Phys. Rev. E 62, R7595 (2000).

[17] G. Monaco, S. Mossa and G. Ruocco, preprint.

[18] W. Petry, E. Bartsch, F. Fujara, M. Kiebel, H. Sillescu and B. Farago, Z. Phys. B 83, 175 (1991).

[19] M. Kiebel, E. Bartsch, O. Debus, F. Fujara, W. Petry and H. Sillescu, Phys. Rev. B 45, 10301 (1992).

[20] D. W. McCall, D. C. Douglass and D. R. Falcone, J. Chem. Phys. 50, 3839 (1969).

[21] F. Fujara, B. Geil, H. Sillescu and G. Fleischer, Z. Phys. B 88, 195 (1992).

[22] J. Wiedersich, T. Blochowicz, S. Benkhof, A. Kudlik, N. V. Surovtsev, C. Tschirwitz, V. N. Novikov and E. Rössler, J. Phys.: Condens. Matter, 11, A147 (1999).

[23] J. Gapinski, W. Steffen, A. Patkowski, A. P. Sokolov, A. Kisliuk, U. Buchenau, M. Russina, F. Mezei and H. Schober, J. Chem. Phys. 110, 2312 (1999).

[24] F. Mezei and M. Russina, J. Phys.: Condens. Matter, 11, A341 (1999).

[25] L. Fabbian, A. Latz, R. Schilling, F. Sciortino, P. Tartaglia, and C. Theis, Phys. Rev. E 60, 5768 (1999); C. Theis, F. Sciortino, A. Latz, R. Schilling, and P. Tartaglia, Phys. Rev. E 62, 1856 (2000); L. Fabbian, A. Latz, R. Schilling, F. Sciortino, P. Tartaglia, and C. Theis, Phys. Rev. E 62, 2388 (2000).

[26] R. Schilling and T. Scheidsteger, Phys. Rev. E 56, 2932 (1997).

[27] P. G. deGennes, Physica (Utrecht) 25,825 (1959).

[28] P. A. Madden, in Liquids, Freezing and the Glass Transition, edited by J. P. Hansen, D. Levesque and J. ZinnJustin (North-Holland, Amsterdam, 1991).

[29] E. Bartsch, H. Bertagnolli, P. Chieux, A. David and H. Sillescu, Chem. Phys. 169, 373 (1993).

[30] G. Monaco, Ph.D. thesis, Università di L'Aquila, 1998.

[31] S. Mossa, Ph.D. thesis, Università di L'Aquila, 1999. 


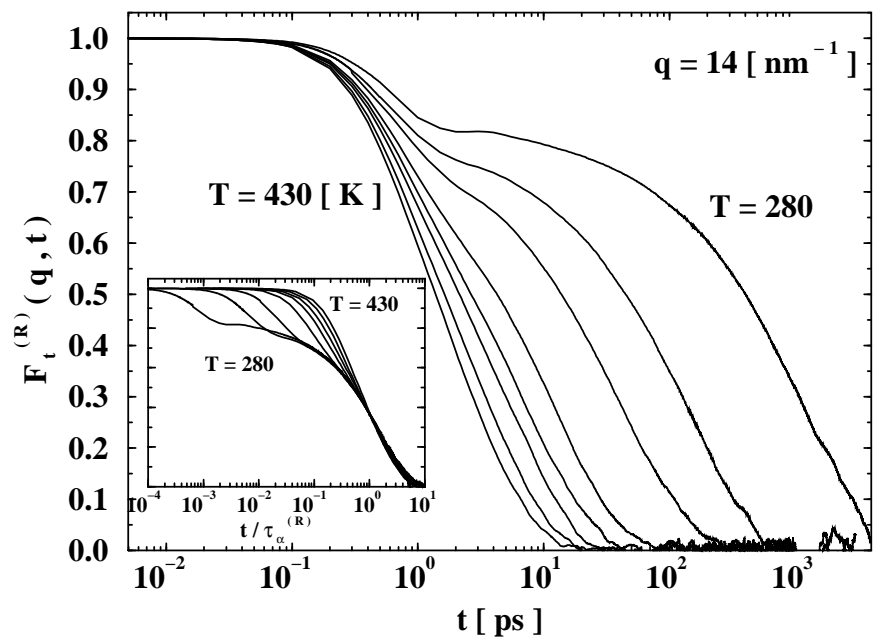

FIG. 1. Intermediate coherent scattering functions $F_{t}^{(R)}(q, t)$ calculated on phenyl rings at $q=14 \mathrm{~nm}^{-1}$ for the temperatures $T=280,300,320,350,370,390,410,430 \mathrm{~K}$ (from top to bottom); in the inset we show the same curves rescaled as a function of $t / \tau_{\alpha}^{(R)}$.

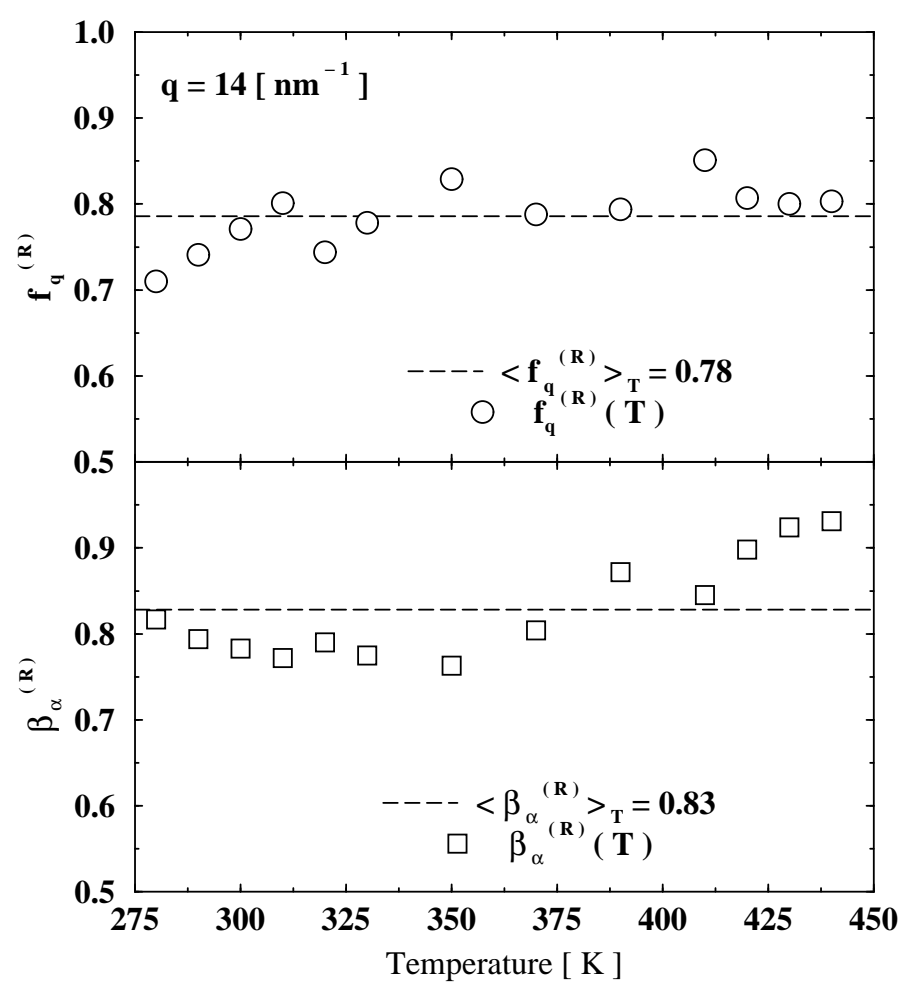

FIG. 2. Temperature dependence of the stretched exponential parameters calculated from $F_{t}^{(R)}(q, t)$ together with the corresponding mean values (dashed lines). Top: non-ergodicity parameter $f_{q}^{(R)}(T)$. Bottom: stretching parameter $\beta_{\alpha}^{(R)}(T)$.

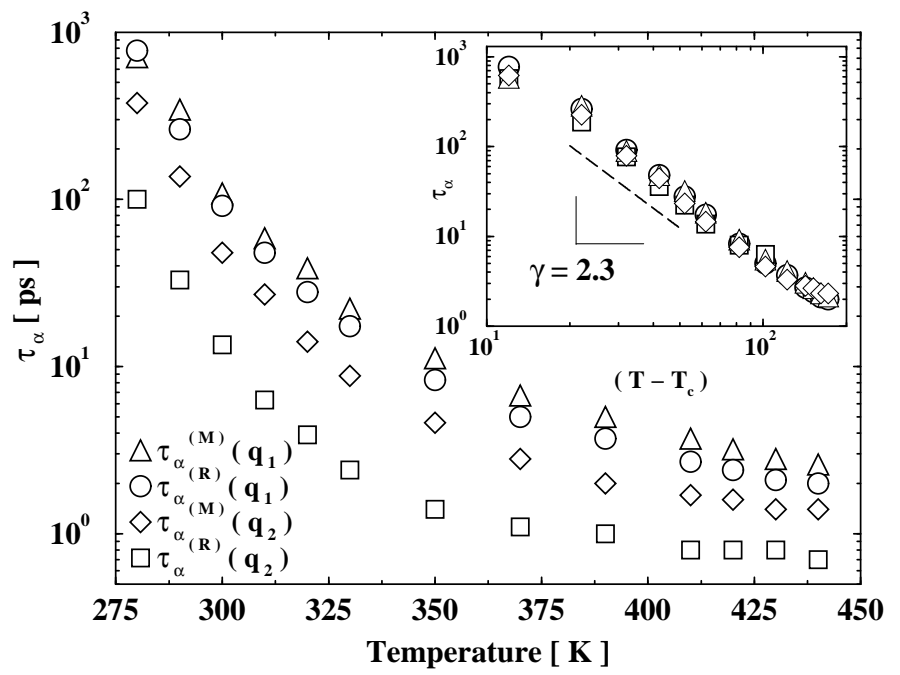

FIG. 3. Temperature dependence of the structural relaxation times at $q_{1}=14 \mathrm{~nm}^{-1}$ and $q_{2}=19 \mathrm{~nm}^{-1}$ calculated both on rings (circles and squares respectively) and molecules (triangles and diamonds respectively) centers of mass. In the inset the date are shown in a double-log scale as a function of the rescaled temperature $\left(T-T_{c}\right)$; the points have been shifted in order to maximize the mutual overlap and to stress the power law behavior. The power law of exponent $\gamma=2.3$ is also shown (dashed line); the value for $T_{c}$ is $268 \mathrm{~K}$.

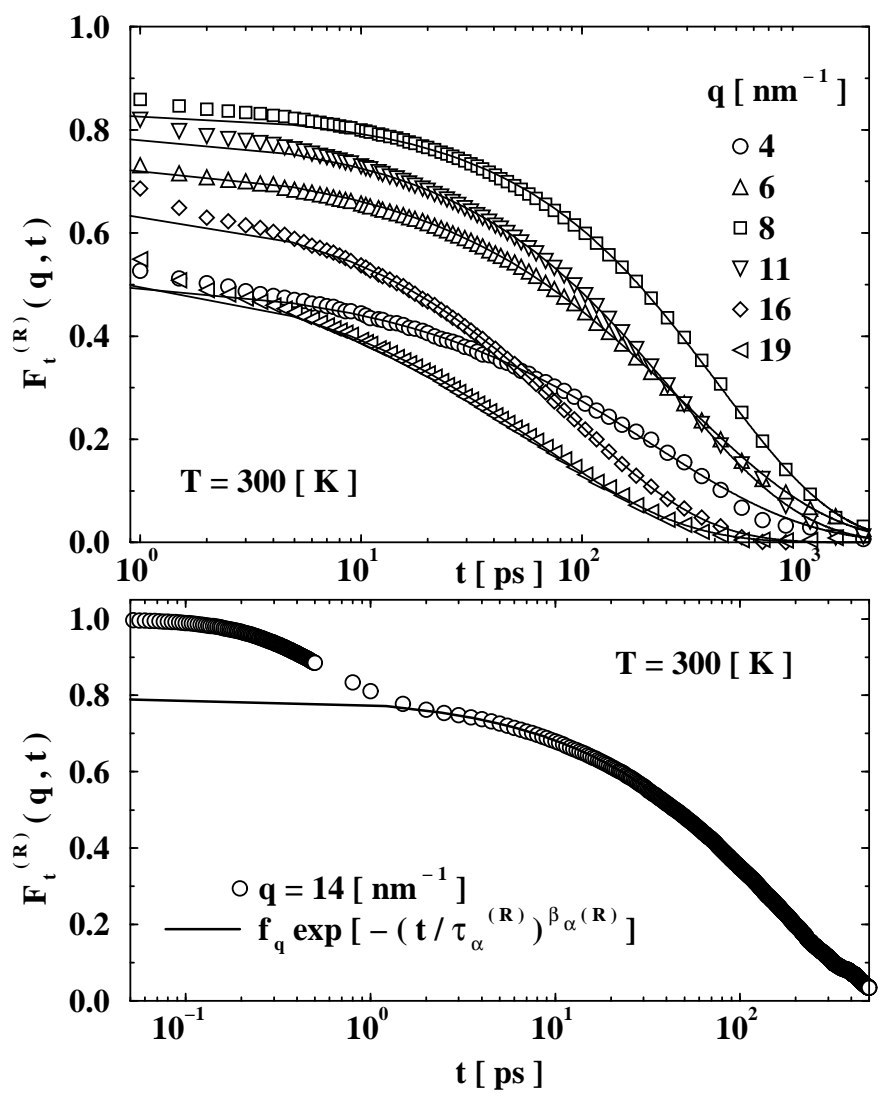


FIG. 4. Top: Intermediate coherent scattering functions $F_{t}^{(R)}(q, t)$ calculated at fixed temperature $T=300 \mathrm{~K}$ for different values of momentum $q$; the corresponding long-time stretched exponential fits are also shown (solid lines). Bottom: Here we show the whole time behavior of $F_{t}^{(R)}(q, t)$ at $q=14 \mathrm{~nm}^{-1}$ together with the stretched exponential fit.

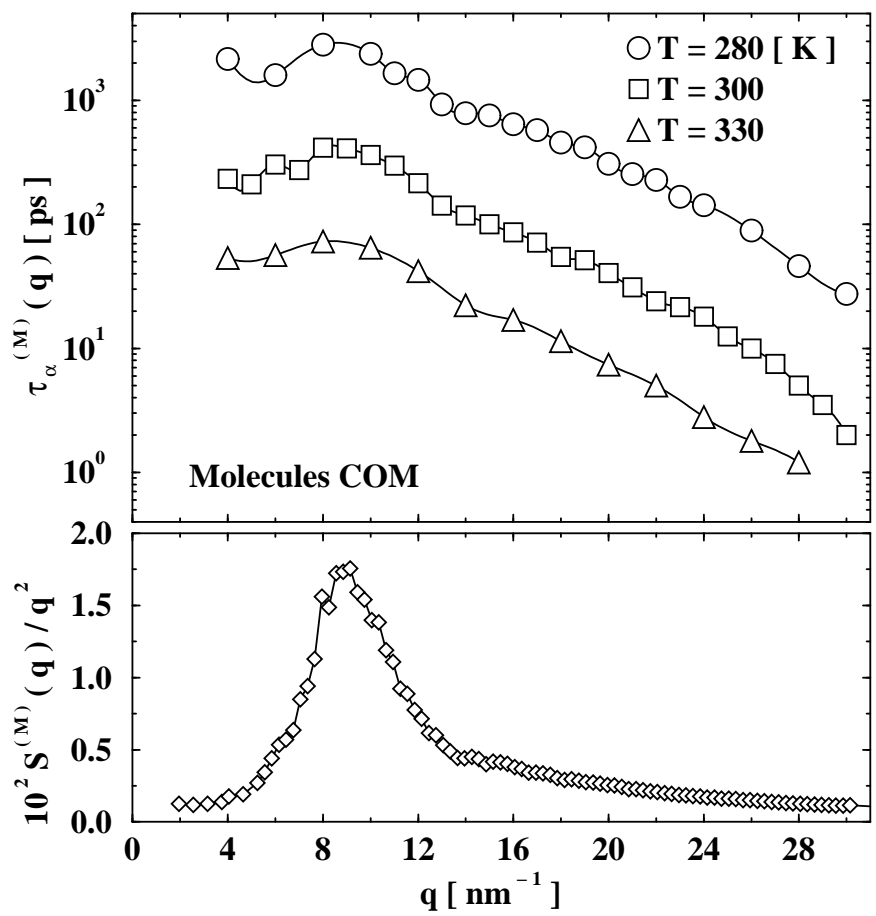

FIG. 5. De Gennes narrowing. Top: Momentum dependence of the structural relaxation time calculated considering molecular centers of mass at the three temperatures $T=280,300,330 \mathrm{~K}$ (circles, squares and triangles respectively). Bottom: Structure factor calculated on molecular centers of masses divided by $q^{2}$.

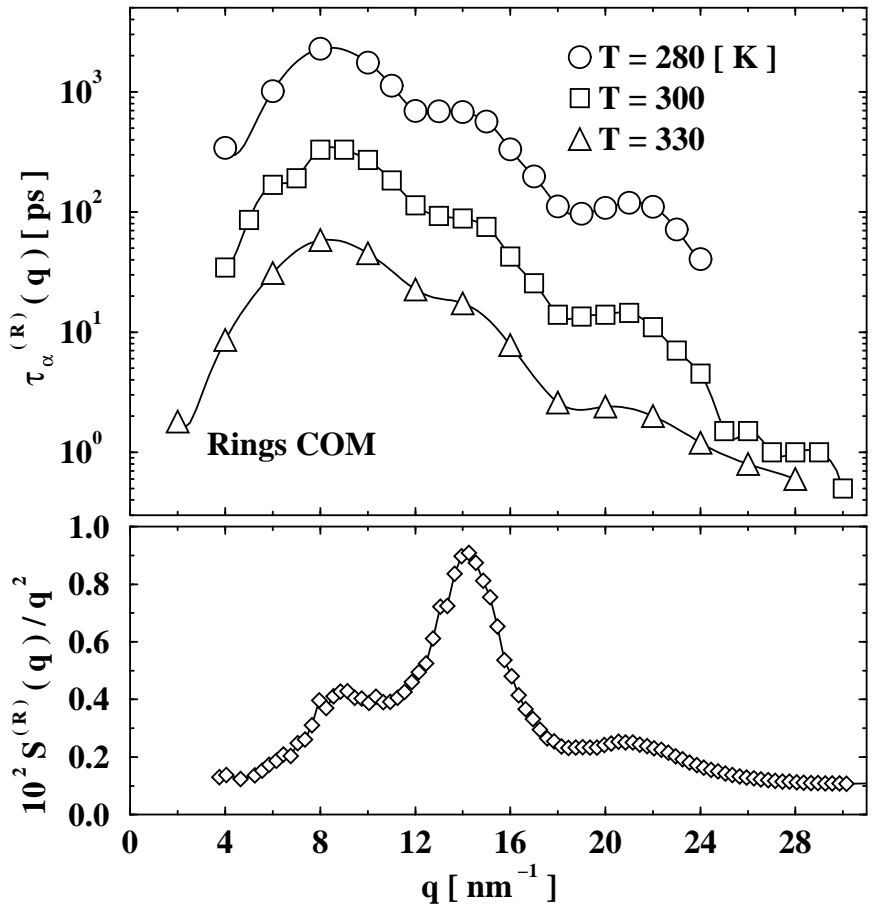

FIG. 6. Top: Momentum dependence of the structural relaxation time calculated considering phenyl rings centers of mass at the three temperatures $T=280,300,330 \mathrm{~K}$ (circles, squares and triangles respectively). Bottom: Structure factor calculated on rings centers of masses divided by $q^{2}$.

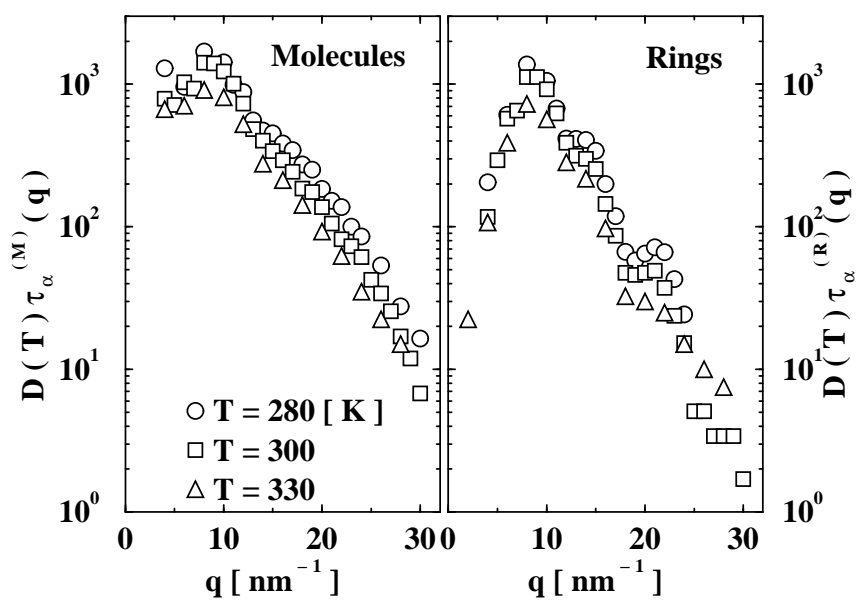

FIG. 7. Left: Structural relaxation times $\tau_{\alpha}^{(M)}$ for molecules at temperatures $T=280,300,330 \mathrm{~K}$ multiplied by the correspondent diffusion coefficients $D(T)$; this product is supposed to be temperature independent. Right: as the left panel but for $\tau_{\alpha}^{(R)}$ calculated on phenyl rings centers of masses. 


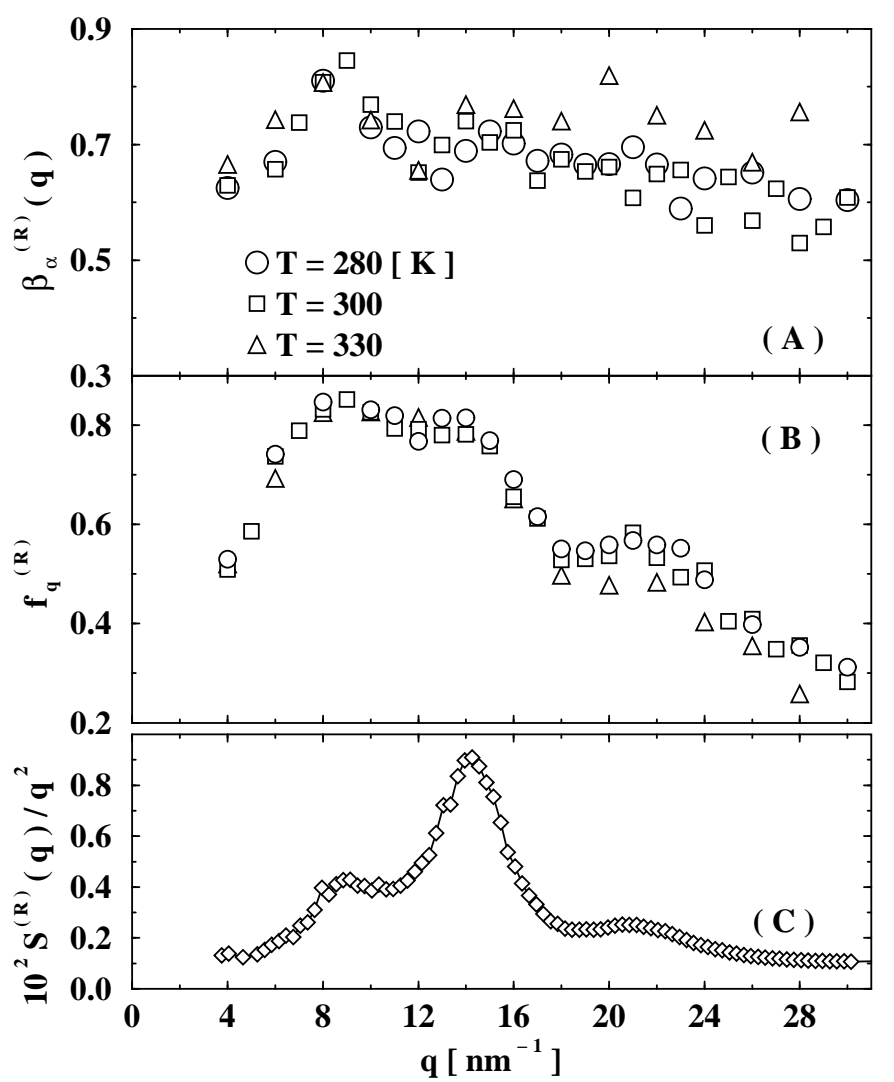

FIG. 8. Momentum dependence of the stretching parameters $\beta_{\alpha}^{(R)}(\mathrm{A})$ and $f_{q}^{(R)}$ (B); oscillations in phase with the structure factor $(\mathrm{C})$ are evident.

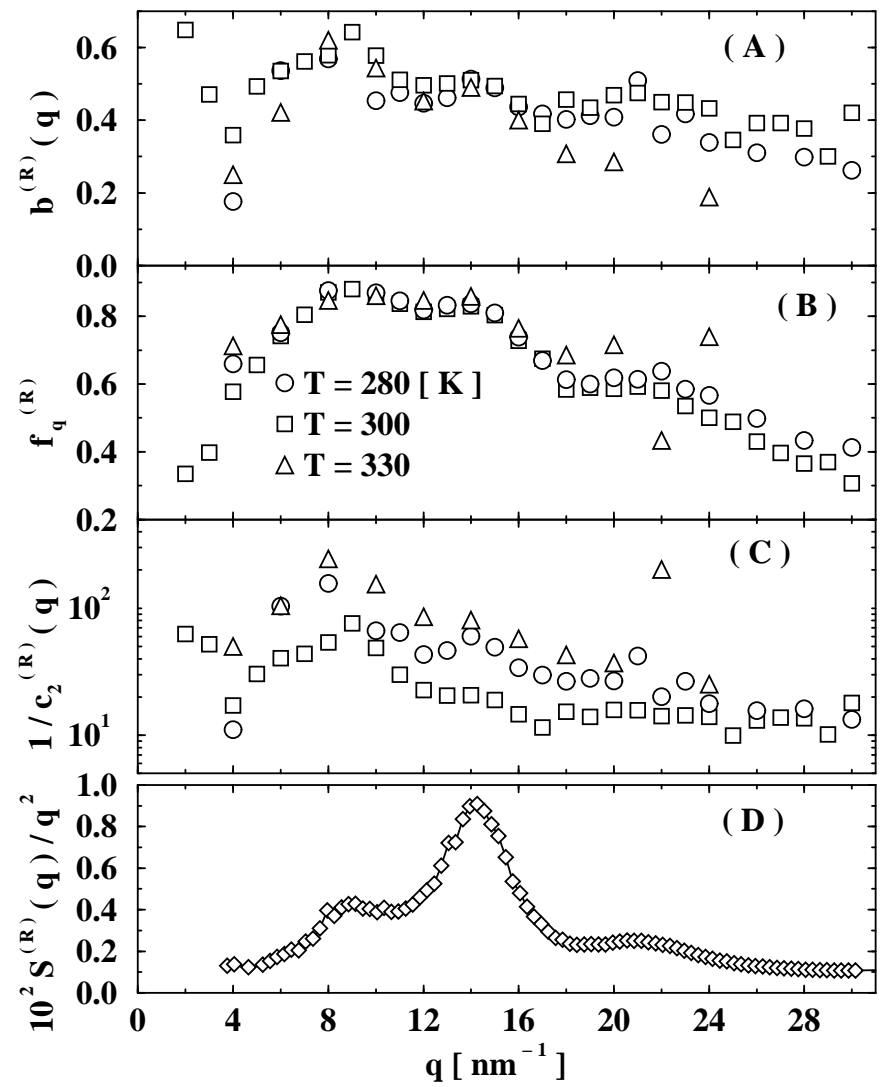

FIG. 9. Momentum dependence of the fitting parameters for the $\beta$-region $b^{(R)}(q)(\mathrm{A}), f_{q}^{(R)}(\mathrm{B})$ and $1 / c_{2}^{(R)}(q)(\mathrm{C})$; also in this case a clear correlation with the static structure factor (D) is evident. 


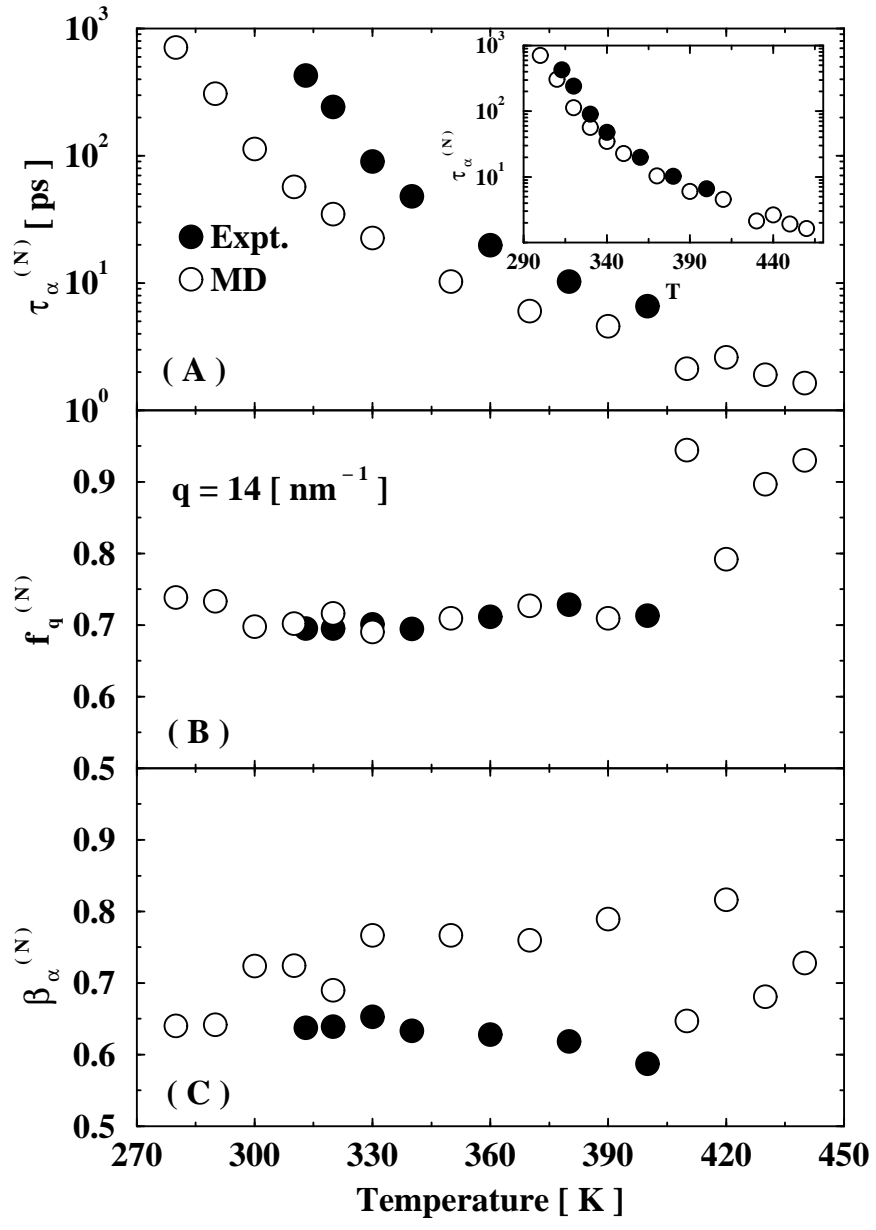

FIG. 10. Comparison among the temperature dependencies of the stretched exponential parameters as calculated by MD simulated neutron spectra (open symbols) and experimental neutron scattering (full symbols) at $q=14 \mathrm{~nm}^{-1}$. (A) relaxation time $\tau_{\alpha}^{(N)}$; in the inset the MD data have been shifted of $20 \mathrm{~K}$ as explained in the text. (B) non ergodicity parameter $f_{q}^{(N)}$. (C) stretching parameter $\beta_{\alpha}^{(N)}$.
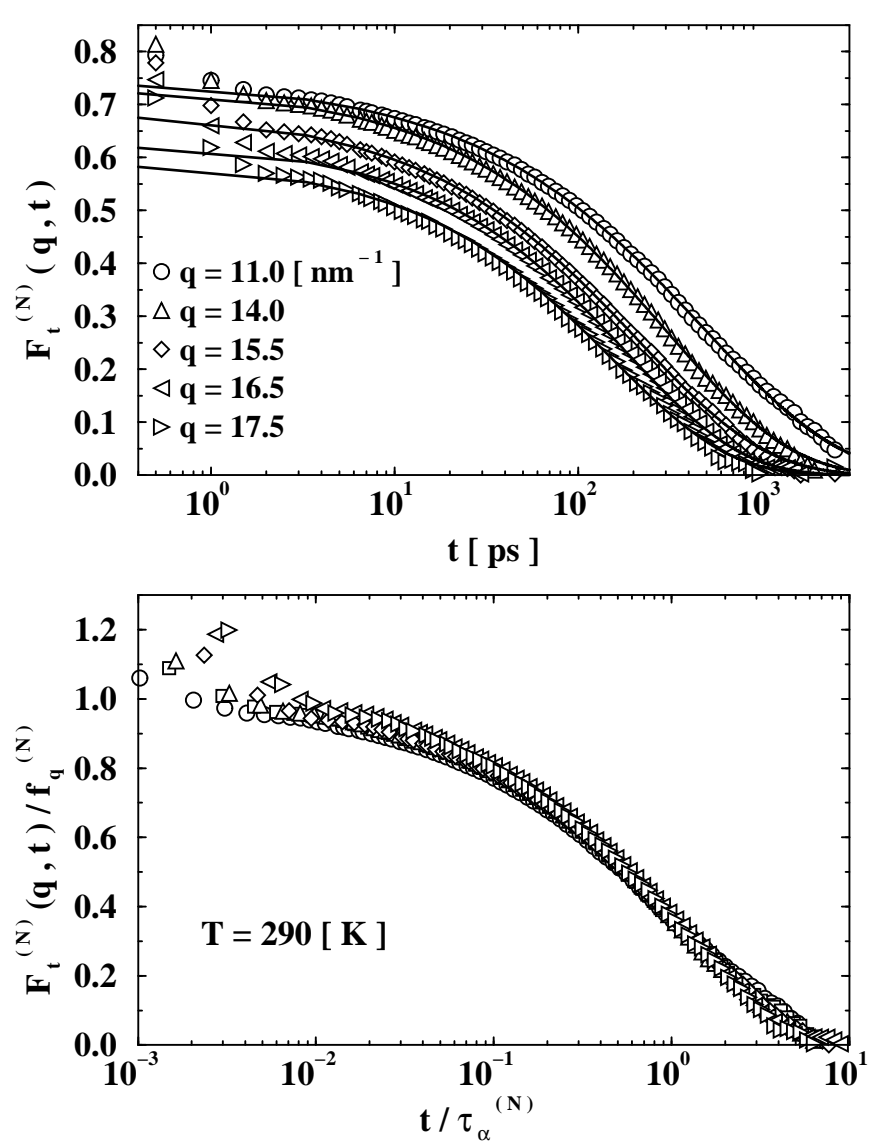

FIG. 11. Top: Simulated neutron coherent scattering function $F_{t}^{(N)}(q, t)$ at $T=290 \mathrm{~K}$ as a function of momentum. Bottom: $F_{t}^{(N)}(q, t) / f_{q}^{(N)}$ plotted as a function of the rescaled time $t / \tau_{\alpha}^{(N)}$. All the curves collapse on a master curve as expected (in this case $f_{q}^{(N)}$ depends on $q$ ).
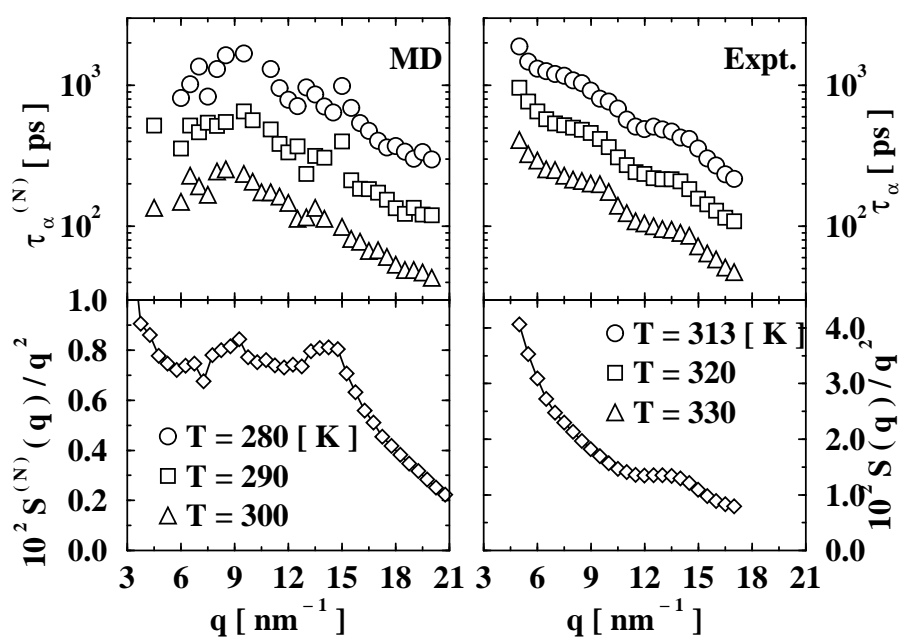

FIG. 12. Comparison between the values of $\tau_{\alpha}^{(N)}$ calculated by MD simulations (left panel) and the experimental ones (right panel) together with the appropriate structure factors divided by $q^{2}$. 


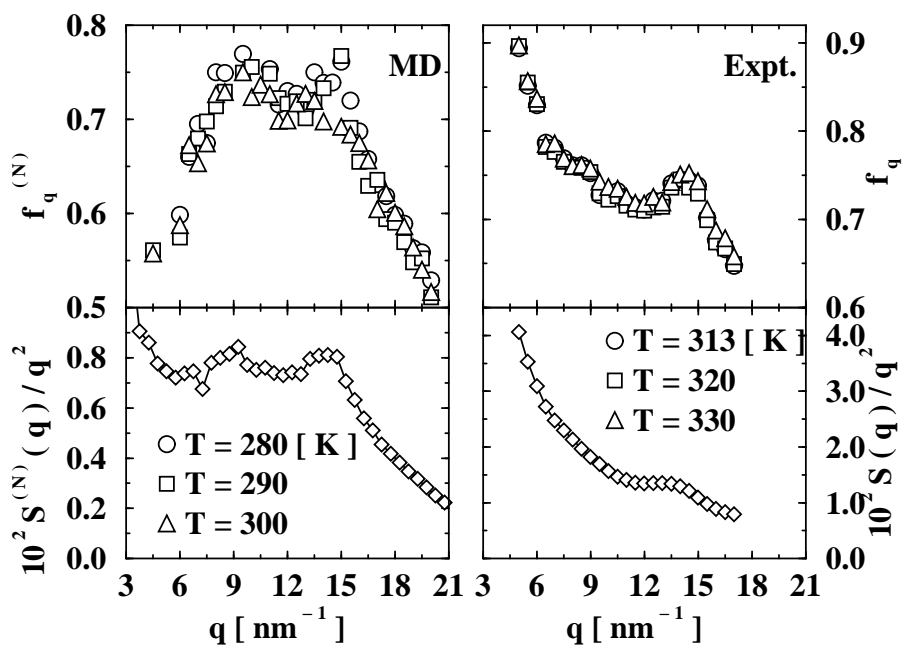

FIG. 13. As above for the non-ergodicity parameter $f_{q}^{(N)}$.

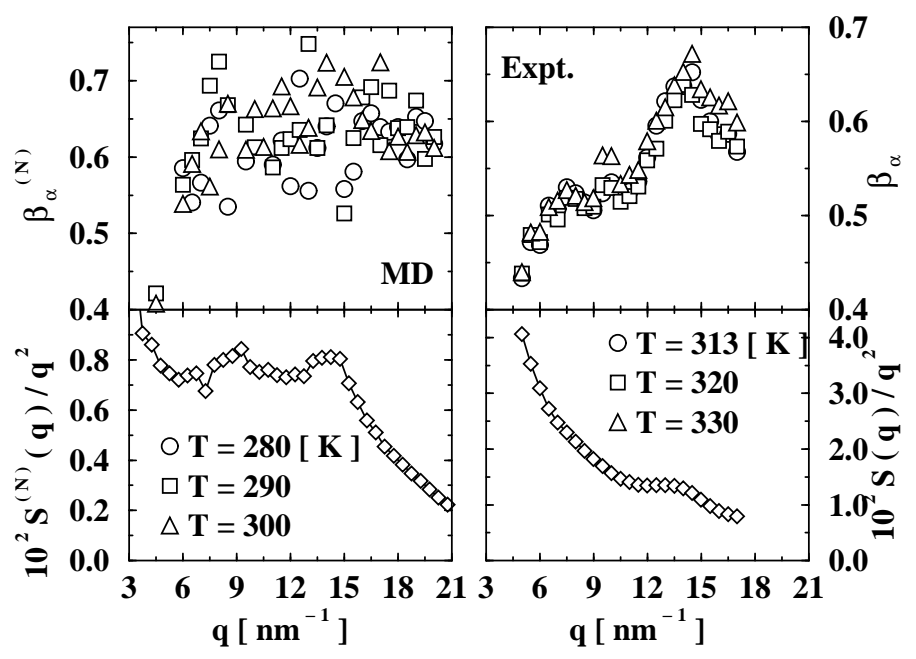

FIG. 14. As above for the stretching factor $\beta_{\alpha}^{(N)}$.

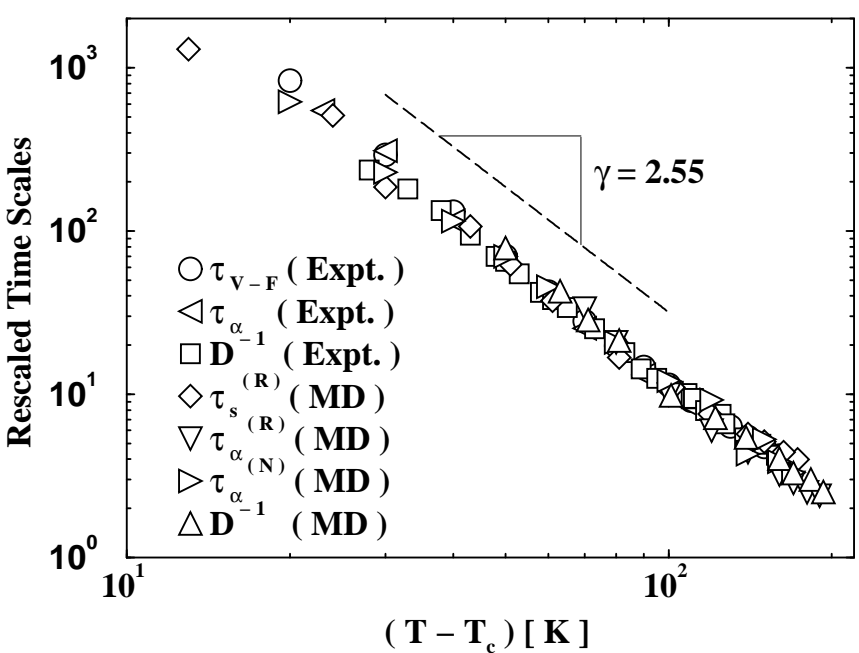

FIG. 15. Master plot of all the time scales related to the center of mass dynamics of the system, calculated by means of molecular dynamics and measured experimentally: experimental viscosity (circles), neutron scattering collective experimental relaxation time (left triangles), inverse of the experimental diffusion coefficient (squares), MD one particle (diamonds) and structural relaxation time (triangles down) calculated on phenyl rings, MD neutron spectra relaxation time (right triangles), inverse of the MD diffusion coefficients (triangles up). The data have been rescaled by arbitrary constants in order to maximize the overlap with the viscosity experimental results. All these time scales follow the same MCT power law with exponents $T_{c}=290 \mathrm{~K}$ and $\gamma=2.55$. 